\title{
Single-cell transcriptomics reveals neuroinflammation in severe COVID-19
}

these data

suggest that

peripheral

inflammation

triggered by

COVID-19 can

still be relayed

to brain cells

\section{considerable research efforts over the}

past year, the mechanisms underlying

these COVID-19-associated

symptoms are still unclear. The aim

of the new study - led by Andrew

C. Yang and Fabian Kern, and supervised by Tony Wyss-Coray and Andreas Keller - was to investigate the molecular changes that occur in the brain during severe COVID- 19.

"It all started with a sense of duty, that we wanted to contribute however we could to pandemic efforts," explains Yang. "The brain is incredibly complex and contains a rich diversity of cell types. So we looked for an unbiased - but also cell type-resolved - approach

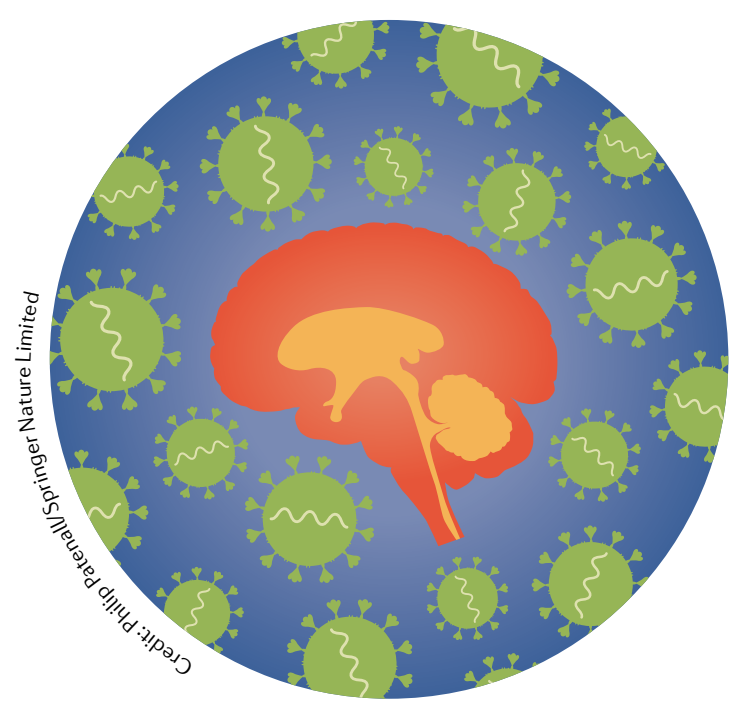

to study how COVID-19 affects the brain, and thought single-cell transcriptomics would be well-suited."

The researchers performed single-nucleus RNA sequencing (snRNA-seq) and immunohistochemistry (IHC) on tissue from a group of individuals who died with COVID-19 and a group of individuals who died from other causes. "Through our collaborators at Saarland University and Stanford University, we had access to well-preserved, frozen post-mortem brain and choroid plexus tissue," notes Yang.

The researchers began by performing snRNA-seq on samples of medial frontal cortex from eight individuals with COVID-19 and eight controls, and used unsupervised clustering to group the nuclei into six broad cell types on the basis of gene expression. In individuals with COVID-19, 786 genes - spread across all six major cell types were expressed differently than in controls. The strongest effect was in astrocytes and other glia, in which transcriptomic changes indicated a COVID-19-associated activation of inflammatory pathways and dysregulation of homeostatic pathways.

Next, Yang and colleagues performed snRNA-seq on choroid plexus samples from seven of the individuals with COVID-19 and a different group of seven controls. Unsupervised clustering identified seven broad cell types, and an upregulation of inflammatory genes was observed in the individuals with COVID-19 compared with controls. IHC also detected higher levels of CD68, a marker of macrophage activation, in the COVID-19 samples.

To establish whether the inflammatory responses resulted from the presence of SARS-CoV-2 in the tissue, Yang and colleagues performed RNA sequencing and RT-qPCR for SARS-CoV-2 RNA on the cortex and choroid plexus samples. No viral RNA was detected in the COVID-19 samples or the controls. The researchers also performed IHC with four different antibodies to SARS-CoV-2. One antibody produced a signal across cortical vasculature, meninges and choroid plexus; however, this signal was not detected with the other three antibodies, leading Yang and colleagues to conclude that they could not specifically detect virus protein in the samples.

"Despite the brain being shielded by the blood-brain barrier, these data suggest that peripheral inflammation triggered by COVID- 19 can still be relayed to brain cells, triggering neuroinflammation", explains Yang.

The researchers have made the snRNA-seq data set publicly available online with the aim of providing a resource for the field.

"Overall, I think our work could offer a molecular mechanism for some of the neurological symptoms that individuals with COVID-19 have reported," concludes Yang. "However, it's important to note that the COVID-19 samples used in this study came from individuals who died with COVID-19, so it's unclear how far we can extrapolate our findings to individuals who recover."

Sarah Lemprière

ORIGINAL ARTICLE Yang, A. C. et al. Dysregulation of brain and choroid plexus cell types in severe COVID-19. Nature https://doi.org/ 10.1038/s41586-021-03710-0 (2021) RELATED ARTICLES Solomon, T.et al. Neurological infection with SARS-CoV-2 - the story so far. Nat. Rev.Neurol. 17, 65-66 (2021) | Pezzini, A. et al. Lifting the mask on neurological manifestations of COVID-19. Nat. Rev. Neurol. 16, 636-644 (2020) 